Mathematical Modelling and Analysis

Volume 21 Number 4, July 2016, 450-465

http://dx.doi.org/10.3846/13926292.2016.1182594

(c) Vilnius Gediminas Technical University, 2016
Publisher: Taylor\&Francis and VGTU

http://www.tandfonline.com/TMMA

ISSN: $1392-6292$

eISSN: $1648-3510$

\title{
Special Splines of Exponential Type for the Solutions of Mass Transfer Problems in Multilayer Domains
}

\author{
Andris Buikis ${ }^{a}$, Harijs Kalis ${ }^{a}$ and Ilmars Kangro ${ }^{b}$ \\ ${ }^{a}$ Institute of Mathematics and Computer Science of University of Latvia \\ Raina bulvāris 29, Rīga LV-1459, Latvija \\ ${ }^{b}$ Rēzekne Higher Education Institution, Departament of Engineering Science \\ Atbrïivoŝanas aleja 90, LV-4601, Rēzekne, Latvija \\ E-mail(corresp.): buikis@latnet. Iv \\ E-mail: kalis@lanet.lv \\ E-mail: kangro@ru.lv
}

Received August 30, 2015; revised April 06, 2016; published online July 1, 2016

\begin{abstract}
We consider averaging methods for solving the 3-D boundary-value problem of second order in multilayer domain.

The special hyperbolic and exponential type splines, with middle integral values of piece-wise smooth function interpolation are considered. With the help of these splines the problems of mathematical physics in 3-D with piece-wise coefficients are reduced with respect to one coordinate to $2-\mathrm{D}$ problems. This procedure also allows to reduce the 2-D problems to 1-D problems and the solution of the approximated problemsa can be obtained analytically. In the case of constant piece-wise coefficients we obtain the exact discrete approximation of a steady-state 1-D boundary-value problem.

The solution of corresponding averaged 3-D initial-boundary value problem is also obtained numerically, using the discretization in space with the central diferences. The approximation of the 3-D nonstationary problem is based on the implicit finite-difference and alternating direction (ADI) methods. The numerical solution is compared with the analytical solution.
\end{abstract}

Keywords: special splines, averaging method, 3D problem, ADI method, analytical solution.

AMS Subject Classification: 65N06; 65N20; 65N40; 65N25; 34C55; 65N35.

\section{Introduction}

The task of sufficient accuracy numerical simulation of quick solution 3-D problems for mathematical physics in multi-layered media is important in the known areas of the applied sciences. With regard to the numerical analysis several numerical methods are known for solving 3-D problems: FEM, BEM, spectral 
methods, multigrids and others methods. An example, in [6] a new template for stability analysis of numerical schemes for parabolic and pseudoparabolic problems with nonlocal conditions is given, in [10] efficient parallel algorithms of the OpenFOAM-based Parallel Solver for simulation of Electrical Power Cables are analysed. For simple engineering calculations two methods are applied [1], [2]: special finite difference scheme and conservative averaging method (CAM) by using special integral hyperbolic type splines with two parameters in every layer. We chose the CAM for engineering calculation and the solution of 3-D problem can be obtained analytically.

CAM by using integral parabolic type splines was developed by A. Buikis in his Doctoral Thesis [3] (in the Russian language) and in several papers [4], [11] etc. as a unified analytical (or analytically-numerical) approach to PDE with discontinuous coefficients. These methods were applied to create the mathematical simulation of the mass transfer 3-D initial-boundary-value problems for parabolic type partial differential equations of second order with piece-wise coefficients in multi-layered underground systems. Domains of a similar composition are examined using other mathematical methods in [5], [8].

In this Paper the special spline with two different functions, which interpolate middle integral values of piece-wise smooth function, is defined. These functions contain the independent solutions of corresponding homogeneous linear ODEs with parameters - characteristic values. Special hyperbolic and exponential type splines are developed. With the help of these splines the problems of mathematical physics in $3-\mathrm{D}$ with piece-wise coefficients with respect to one coordinate are reduced to problems for system of PDEs in 2-D.

The solutions of corresponding 3-D initial-boundary value problem in one layer are obtained numerically, using the implicit alternating method by Duglas and Rackford (ADI) [7]. The 3-D problem in multi-layered domain is reduced to 2-D and 1-D problems, using special integral exponential - type splines. Every layer of these splines contain parameters, which can be selected in order to decrease the error of the solution. In the unlikely event if the parameters for the hyperbolic type spline tend to zero, we get the integral parabolic spline, obtained from A.Buikis.

\section{Formulation of Problem}

The process of diffusion and convection is considered in a 3-D parallelepiped

$$
\Omega=\left\{(x, y, z): 0 \leq x \leq L_{x}, 0 \leq y \leq L_{y}, 0 \leq z \leq L_{z}\right\} .
$$

The domain $\Omega$ consist of multilayer medium.

We will consider the nonstationary $3-\mathrm{D}$ problem for multi-layered piece-wise homogenous materials of $\mathrm{N}$ layers in the domain

$$
\Omega_{i}=\left\{(x, y, z): x \in\left(0, L_{x}\right), y \in\left(0, L_{y}\right), z \in\left(z_{i-1}, z_{i}\right)\right\}, i=\overline{1, N},
$$

where $H_{i}=z_{i}-z_{i-1}$ is the height of layer $\Omega_{i}, z_{0}=0, z_{N}=L_{z}$.

We will find the distribution of mass transfer $c_{i}=c_{i}(x, y, z, t)$ in every layer $\Omega_{i}$ at the point $(x, y, z) \in \Omega_{i}$ and at the time $\mathrm{t}$ by solving the following 3 $\mathrm{D}$ initial-boundary value problem for partial differential equation of parabolic 
type (PDE) [8]:

$$
\left\{\begin{array}{l}
\frac{\partial c_{i}}{\partial t}=\frac{\partial}{\partial x}\left(D_{i x} \frac{\partial c_{i}}{\partial x}\right)+r_{i x} \frac{\partial c_{i}}{\partial x}+\frac{\partial}{\partial y}\left(D_{i y} \frac{\partial c_{i}}{\partial y}\right)+r_{i y} \frac{\partial c_{i}}{\partial y} \\
\quad+\frac{\partial}{\partial z}\left(D_{i z} \frac{\partial c_{i}}{\partial z}\right)+r_{i z} \frac{\partial c_{i}}{\partial z}-a_{i 0}^{2} c_{i}+f_{i}, \\
x \in\left(0, L_{x}\right), y \in\left(0, L_{y}\right), z \in\left(z_{i-1}, z_{i}\right), t \in\left(0, t_{f}\right), i=\overline{1, N}, \\
\frac{\partial c_{i}(0, y, z, t)}{\partial x}=\frac{\partial c_{i}(x, 0, z, t)}{\partial y}=0, \\
D_{1 z} \frac{\partial c_{1}(x, y, 0, t)}{\partial z}-\beta_{z}\left(c_{1}(x, y, 0, t)-c_{o z}(x, y)\right)=0, \\
D_{i x} \frac{\partial c_{i}\left(L_{x}, y, z, t\right)}{\partial x}+\alpha_{i x}\left(c_{i}\left(L_{x}, y, z, t\right)-c_{i a x}(y, z)\right)=0, i=\overline{1, N} \\
D_{i y} \frac{\partial c_{i}\left(x, L_{y}, z, t\right)}{\partial y}+\alpha_{i y}\left(c_{i}\left(x, L_{y}, z, t\right)-c_{i a y}(x, z)\right)=0, i=\overline{1, N} \\
D_{N z} \frac{\partial c_{N}\left(x, y, L_{z}, t\right)}{\partial z}+\alpha_{z}\left(c_{N}\left(x, y, L_{z}, t\right)-c_{a z}(x, y)\right)=0, \\
c_{i}\left(x, y, z_{i}, t\right)=c_{i+1}\left(x, y, z_{i}, t\right), \\
D_{i z} \frac{\partial c_{i}\left(x, y, z_{i}, t\right)}{\partial z}=D_{i+1, z} \frac{\partial c_{i+1}\left(x, y, z_{1}, t\right)}{\partial z}, i=\overline{1, N-1} \\
c_{i}(x, y, z, 0)=c_{i 0}(x, y, z), i=\overline{1, N},
\end{array}\right.
$$

where $c_{i}=c_{i}(x, y, z, t)$ are the solutions in every layer, $f_{i}=f_{i}(x, y, z, t)$ are the fixed sours functions, $D_{i x}, D_{i y}, D_{i z}, r_{i x}, r_{i y}, r_{i z}, a_{i 0}$ are the constant coefficients, $\alpha_{i x}, \alpha_{i y}, \alpha_{z}, \beta_{z}, i=\overline{1, N}$ are the constant mass transfer coefficients in the third kind boundary conditions, $c_{a z}, c_{i a y}, c_{i a x}, c_{o z}$ are the given concentration on the boundary, $t_{f}$ is the final time, $c_{i 0}(x, y, z)$ are the given initial concentration.

\section{The averaging method in $z$-direction using the special integral spline with two fixed functions}

By using the averaging method with respect to $z$ with two fixed parametrical functions $f_{i z 1}, f_{i z 2}, i=\overline{1, N}$ we construct a spline

$$
c_{i}(x, y, z, t)=c_{i z}(x, y, t)+m_{i z}(x, y, t) f_{i z 1}\left(z-\overline{z_{i}}\right)+e_{i z} G_{i z} f_{i z 2}\left(z-\overline{z_{i}}\right),
$$

where $c_{i z}(x, y, t)=\frac{1}{H_{i}} \int_{z_{i-1}}^{z_{i}} c_{i}(x, y, z, t) d z, G_{i z}=H_{i} / D_{i z}$,

$$
\int_{z_{i-1}}^{z_{i}} f_{i z 1} d z=\int_{z_{i-1}}^{z_{i}} f_{i z 2} d z=0, \overline{z_{i}}=\left(z_{i-1}+z_{i}\right) / 2, z \in\left[z_{i-1}, z_{i}\right], i=\overline{1, N} .
$$

For example, using the exponential functions $f_{i z 1}$ and $f_{i z 2}$ can be defined in the following way:

$$
\begin{aligned}
& f_{i z 1}=\exp \left(a_{i z 1}\left(z-\overline{z_{i}}\right)\right)-\frac{2}{a_{i z 1} H_{i}} \sinh \left(0.5 a_{i z 1} H_{i}\right), \\
& f_{i z 2}=\exp \left(a_{i z 2}\left(z-\overline{z_{i}}\right)\right)-\frac{2}{a_{i z 2} H_{i}} \sinh \left(0.5 a_{i z 2} H_{i}\right),
\end{aligned}
$$

where $a_{i z 1}=-\frac{r_{i z}}{2 D_{i z}}-\sqrt{\frac{r_{i z}^{2}}{4 D_{i z}^{2}}+\frac{a_{i 0}^{2}}{D_{i z}}}, a_{i z 2}=-\frac{r_{i z}}{2 D_{i z}}+\sqrt{\frac{r_{i z}^{2}}{4 D_{i z}^{2}}+\frac{a_{i 0}^{2}}{D_{i z}}}$. 
We can determine the unknown functions $m_{i z}(x, y, t), e_{i z}(x, y, t)$ from boundary conditions (2.1) in z-direction:

1) $m_{1 z} a_{11}^{m}+G_{1 z} e_{1 z} a_{12}^{m}=\beta_{z}\left(c_{1 z}-c_{0 z}\right)$, for $z=0$,

2) $D_{N z}\left(m_{N z} f_{N z 1}^{a p}+G_{N z} e_{N z} f_{N z 2}^{a p}\right)+\alpha_{z}\left(c_{N z}-c_{a z}\right.$

$$
\left.+m_{N z} f_{N z 1}^{p}+e_{N z} G_{N z} f_{N z 2}^{p}\right)=0, \quad \text { for } z=L_{z},
$$

3) $c_{i z}+m_{i z} f_{i z 1}^{p}+e_{i z} G_{i z} f_{i z 2}^{p}=c_{i+1, z}+m_{i+1, z} f_{i+1, z 1}^{m}+e_{i+1, z} G_{i+1, z} f_{i+1, z 2}^{m}$,

$$
\begin{aligned}
& D_{i z}\left(m_{i z} f_{i z 1}^{a p}+e_{i z} G_{i z} f_{i z 2}^{a p}\right)=D_{i+1, z}\left(m_{i+1, z} f_{i+1, z 1}^{a m}+e_{i+1, z} G_{i+1, z} f_{i+1, z 2}^{a m}\right), \\
& \quad \text { for } z=z_{i}, \quad i=\overline{1, N-1},
\end{aligned}
$$

where $f_{i z k}^{m}=f_{i z k}\left(-0.5 H_{i}\right), f_{i z k}^{p}=f_{i z k}\left(0.5 H_{i}\right), f_{i z k}^{a m}=\frac{\partial f_{i z k}}{\partial z}\left(-0.5 H_{i}\right), f_{i z k}^{a p}=$ $\frac{\partial f_{i z k}}{\partial z}\left(0.5 H_{i}\right), k=1 ; 2, a_{11}^{m}=D_{1 z} f_{1 z 1}^{a m}-\beta_{z} f_{1 z 1}^{m}, a_{12}^{m}=D_{1 z} f_{1 z 2}^{a m}-\beta_{z} f_{1 z 2}^{m}$.

For determination of $e_{i z}$, from conditions on the contact lines $z=z_{i}, i=$ $\overline{1, N-1}, z=z_{i-1}, i=\overline{2, N}$ by excluding $m_{i+1, z}, m_{i-1, z}$ and $m_{i z}$ we obtain the following system of $N-2$ algebraic equations

$$
\begin{gathered}
a_{i, i} e_{i z}+a_{i, i+1} e_{i+1, z}+a_{i, i-1} e_{i-1, z}=b_{i, i} c_{i z}+b_{i, i+1} c_{i+1, z} \\
+b_{i, i-1} c_{i-1, z}, \quad i=\overline{2, N-1}
\end{gathered}
$$

where $b_{i, i+1}=-c_{i 1}^{m}, b_{i, i-1}=-c_{i 1}^{p}, b_{i, i}=-\left(b_{i, i+1}+b_{i, i-1}\right), a_{i, i}=G_{i z}\left(b_{i, i+1} c_{i 2}^{p}+\right.$ $\left.b_{i, i-1} c_{i 2}^{m}\right), a_{i, i+1}=G_{i+1, z} b_{i, i+1} c_{i 3}^{p}, a_{i, i-1}=G_{i-1, z} c_{i 1}^{p} c_{i 3}^{m}, c_{i 1}^{p}=f_{i z 1}^{p}-\kappa_{i z 1} f_{i+1, z 1}^{m}$, $c_{i 2}^{p}=f_{i z 2}^{p}-\kappa_{i z 2} f_{i+1, z 1}^{m}, c_{i 3}^{p}=\delta_{i+1, z}^{m} f_{i+1, z 1}^{m}-f_{i+1, z 2}^{m}, \kappa_{i z 1}=\frac{D_{i z} f_{i z 1}^{a p}}{D_{i+1, z} f_{i+1, z 1}^{a m}}, \kappa_{i z 3}=$ $\frac{D_{i z} f_{i z 2}^{a m}}{D_{i-1, z} f_{i-1, z 1}^{a p}}, \kappa_{i z 2}=\frac{D_{i z} f_{i z 2}^{a p}}{D_{i+1, z} f_{i+1, z 1}^{a m}}, \delta_{i+1, z}^{m}=\frac{f_{i+1, z 2}^{a m}}{f_{i+1, z 1}^{a m}}, c_{i 1}^{m}=\kappa_{i-1, z 1}^{-1} f_{i-1, z 1}^{p}-f_{i z 1}^{m}$, $c_{i 2}^{m}=f_{i z 2}^{m}-\kappa_{i, z 3} f_{i-1, z 1}^{p}, c_{i 3}^{m}=f_{i-1, z 2}^{p}-\delta_{i-1, z}^{p} f_{i-1, z 1}^{p}, \delta_{i-1, z}^{p}=f_{i-1, z 2}^{a p} / f_{i-1, z 1}^{a p}$.

From the boundary conditions at $z=0, z=L_{z}$ and previous expressions for $i=1$ and $i=N$ by excluding $m_{1 z}, m_{N z}$ we get:

$$
\begin{aligned}
& a_{1,1} e_{1 z}+a_{1,2} e_{2 z}=b_{1,1} c_{1 z}+b_{1,2} c_{2 z}+b_{10} c_{0 z}, \\
& a_{N, N} e_{N z}+a_{N, N-1} e_{N-1, z}=b_{N, N} c_{N z}+b_{N, N-1} c_{N-1, z}+b_{N, 0} c_{a z},
\end{aligned}
$$

where $a_{1,1}=G_{1 z}\left(c_{12}^{p} a_{11}^{m}-c_{11}^{p} a_{12}^{m}\right), a_{1,2}=G_{2, z} c_{13}^{p} a_{11}^{m}, b_{1,1}=-a_{11}^{m}-\beta_{z} c_{11}^{p}, b_{1,2}=$ $a_{11}^{m}, b_{10}=\beta_{z} c_{11}^{p}, a_{N, N}=-G_{N z}\left(c_{N 1}^{m} a_{12}^{p}+c_{N 2}^{m} a_{11}^{p}\right), a_{N, N-1}=G_{N-1, z} c_{N 3}^{m} a_{11}^{p}$, $b_{N, N}=\alpha_{z} c_{N 1}^{m}+a_{11}^{p}, b_{N, N-1}=-a_{11}^{p}, b_{N, 0}=-\alpha_{z} c_{N 1}^{m}, a_{11}^{p}=D_{N z} f_{N z 1}^{a p}+\alpha_{z} f_{N z 1}^{p}$, $a_{12}^{p}=D_{N z} f_{N z 2}^{a p}+\alpha_{z} f_{N z 2}^{p}$.

Using the obtained values $a_{i, j}, b_{i, j}, i, j=\overline{1, N}$ we can determine the 3 diagonal $N$-order matrices $A_{z}^{e}, B_{z}^{e}$ with these elements, the $N$-order diagonal matrix $B_{0}$ with the elements $\left[b_{10}, 0,0, \ldots 0, b_{N, 0}\right]$, the $N$-order vectors-column $e_{z}, c_{z}$ with the elements $e_{i z}, c_{i z}$ and the $N$-order vector-column $c_{0}$ with the elements $\left[c_{0 z}, 0,0, \ldots 0, c_{a z}\right]$. Then we have the system of $N$ algebraic equations in the following vector form

$$
A_{z}^{e} e_{z}(x, y, t)=B_{z}^{e} c_{z}(x, y, t)+B_{0} c_{0}(x, y)
$$

The matrix $A_{z}^{e}$ is a diagonal dominant and we can write the unique solution in the form $e_{z}(x, y, t)=B_{1 z}^{e} c_{z}(x, y, t)+B_{2 z}^{e} c_{0}(x, y)$, where $B_{1 z}^{e}=\left(A_{z}^{e}\right)^{-1} B_{z}^{e}, B_{2 z}^{e}=$ 
$\left(A_{z}^{e}\right)^{-1} B_{0}$. Similarly we obtain $m_{z}(x, y, t)=B_{1 z}^{m} c_{z}(x, y, t)+B_{2 z}^{m} c_{0}(x, y)$, where $B_{1 z}^{m}=B_{z}^{m} B_{1 z}^{e}+C_{z}^{m}, B_{2 z}^{m}=B_{z}^{m} B_{2 z}^{e}+C_{0}^{m}$. Here $B_{z}^{m}, C_{z}^{m}$ are the 2-diagonal N-order matrices with the elements $b_{1,1}^{m}=-\frac{G_{1 z} a_{12}^{m}}{a_{11}^{m}}, b_{i, i-1}^{m}=-\frac{G_{i-1, z} c_{i 3}^{m}}{c_{i 1}^{m}}, b_{i, i}^{m}=$ $\frac{G_{i, z} c_{i 2}^{m}}{c_{i 1}^{m}}, c_{1,1}^{m}=\frac{\beta_{z}}{a_{11}^{m}}, c_{i, i}^{m}=1 / c_{i 1}^{m}, c_{i, i-1}^{m}=-1 / c_{i 1}^{m}, C_{0}^{m}$ is the N-order diagonal matrix with the elements $\left[-\beta_{z} / a_{11}^{m}, 0,0, \ldots 0,0\right]$.

We can determine the diagonal N-order matrices $D_{x}, D_{y}, r_{x}, r_{y}, \alpha_{x}, \alpha_{y}, H_{z}^{e}$, $H_{z}^{m}, a_{0}^{2}$ with the corresponding elements $D_{i x}, D_{i y}, r_{i x}, r_{i y}, \alpha_{i x}, \alpha_{i y}, h_{i z}^{e}, h_{i z}^{m}$, $a_{i 0}^{2}$ and the N-order vectors-columns $f_{z}(x, y, t), c_{a y}^{v}(x), c_{a x}^{v}(y), c_{z, 0}, c_{z}$ with the elements $f_{i z}(x, y, t), c_{i a y}^{v}(x), c_{i a x}^{v}(y), c_{i z, 0}(x, y), c_{i z}(x, y, t)$, where $h_{i z}^{e}=$ $\frac{G_{i z}}{H_{i}}\left(D_{i z}\left(f_{i z 2}^{a p}-f_{i z 2}^{a m}\right)+r_{i z}\left(f_{i z 2}^{p}-f_{i z 2}^{m}\right)\right), h_{i z}^{m}=\frac{1}{H_{i}}\left(D_{i z}\left(f_{i z 1}^{a p}-f_{i z 1}^{a m}\right)+r_{i z}\left(f_{i z 1}^{p}-\right.\right.$ $\left.\left.f_{i z 1}^{m}\right)\right), f_{i z}(x, y, t)=\frac{1}{H_{i}} \int_{z_{i-1}}^{z_{i}} f_{i}(x, y, z, t) d z, c_{i a x}^{v}(y)=\frac{1}{H_{i}} \int_{z_{i-1}}^{z_{i}} c_{i a x}(y, z) d z$, $c_{i a y}^{v}(x)=\frac{1}{H_{i}} \int_{z_{i-1}}^{z_{i}} c_{i a y}(x, z) d z, c_{i z, 0}(x, y)=\frac{1}{H_{i}} \int_{z_{i-1}}^{z_{i}} c_{i 0}(x, y, z) d z$.

Then we have the new initial-boundary value $2 \mathrm{D}$ problem in following vector form:

$$
\left\{\begin{array}{l}
\frac{\partial c_{z}(x, y, t)}{\partial t}=\frac{\partial}{\partial x}\left(D_{x} \frac{\partial c_{z}(x, y, t)}{\partial x}\right)+r_{x} \frac{\partial c_{z}(x, y, t)}{\partial x}+\frac{\partial}{\partial y}\left(D_{y} \frac{\partial c_{z}(x, y, t)}{\partial y}\right) \\
\quad+r_{y} \frac{\partial c_{z}(x, y, t)}{\partial y}-a_{0}^{2} c_{z}(x, y, t)+\left(D h_{z}^{e} B_{1 z}^{e}+D h_{z}^{m} B_{1 z}^{m}\right) c_{z}(x, y, t) \\
\quad+\left(D h_{z}^{e} B_{2 z}^{e}+D h_{z}^{m} B_{2 z}^{m}\right) c_{0}(x, y)+f_{z}(x, y, t) \\
\frac{\partial c_{z}(0, y, t)}{\partial x}=\frac{\partial c_{z}(x, 0, t)}{\partial y}=0 \\
D_{x} \frac{\partial c_{z}\left(L_{x}, y, t\right)}{\partial x}+\alpha_{x}\left(c_{z}\left(L_{x}, y, t\right)-c_{a x}^{v}(y)\right)=0 \\
D_{y} \frac{\partial c_{z}\left(x, L_{y}, t\right)}{\partial y}+\alpha_{y}\left(c_{z}\left(x, L_{y}, t\right)-c_{a y}^{v}(x)\right)=0 \\
c_{z}(x, y, 0)=c_{z, 0}(x, y), x \in\left(0, L_{x}\right), y \in\left(0, L_{y}\right), t \in\left(0, t_{f}\right) .
\end{array}\right.
$$

\section{The averaging method in $y$-direction}

Using the averaging method with respect to $y$ with two fixed parametrical functions $f_{i y 1}, f_{i y 2}, i=\overline{1, N}$ :

$$
c_{i z}(x, y, t)=c_{i y}(x, t)+m_{i y}(x, t) f_{i y 1}\left(y-0.5 L_{y}\right)+e_{i y}(x, t) G_{i y} f_{i y 2}\left(y-0.5 L_{y}\right),
$$

where $c_{i y}(x, t)=\frac{1}{L_{y}} \int_{0}^{L_{y}} c_{i z}(x, y, t) d y, G_{i y}=\frac{L_{y}}{D_{i y}}, \int_{0}^{L_{y}} f_{i y 1} d y=\int_{0}^{L_{y}} f_{i y 2} d y=$ $0, i=\overline{1, N}$. For example, $f_{i y 1}=\exp \left(a_{i y 1}\left(y-0.5 L_{y}\right)\right)-\frac{2}{a_{i y 1} L_{y}} \sinh \left(0.5 a_{i y 1} L_{y}\right)$, $f_{i y 2}=\exp \left(a_{i y 2}\left(y-0.5 L_{y}\right)\right)-\frac{2}{a_{i y 2} L_{y}} \sinh \left(0.5 a_{i y 2} L_{y}\right)$, where $a_{i y 1}=-\frac{r_{i y}}{2 D_{i y}}-$ $\sqrt{\frac{r_{i y}^{2}}{4 D_{i y}^{2}}+\frac{k_{i y 0}^{2}}{D_{i y}}}, a_{i y 2}=-\frac{r_{i y}}{2 D_{i y}}+\sqrt{\frac{r_{i y}^{2}}{4 D_{i y}^{2}}+\frac{k_{i y 0}^{2}}{D_{i y}}}, k_{i y 0}^{2}=a_{i 0}^{2}-a_{i y}, a_{i y} \leq 0$ is the coefficient on $c_{i z}$ in equations (3.2).

We can determine the unknown functions $m_{i y}(x, t), e_{i y}(x, t)$, from boundary conditions (3.2) in the following form:

$$
\begin{aligned}
& D_{i y}\left(m_{i y} f_{i y 1}^{a p}+e_{i y} G_{i y} f_{i y 2}^{a p}\right)+\alpha_{i y}\left(m_{i y} f_{i y 1}^{p}+e_{i y} G_{i y} f_{i y 2}^{p}+c_{i y}(x, t)-c_{i a y}^{v}(x)\right)=0, \\
& m_{i y} f_{i y 1}^{a m}+e_{i y} G_{i y} f_{i y 2}^{a m}=0 \text { or } e_{i y}=-b_{i y}\left(c_{i y}(x, t)-c_{i a y}^{v}(x)\right), m_{i y}=-G_{i y} \kappa_{i y}^{m} e_{i y},
\end{aligned}
$$


where $b_{i y}=\alpha_{i y} /\left(\alpha_{i y} G_{i y}\left(f_{i y 2}^{p}-\kappa_{i y}^{m} f_{i y 1}^{p}\right)+L_{y}\left(f_{i y 2}^{a p}-\kappa_{i y}^{m} f_{i y 1}^{a p}\right)\right), \kappa_{i y}^{m}=\frac{f_{i y 2}^{a m}}{f_{i y 1}^{a m}}, f_{i y k}^{m}=$ $f_{i y k}\left(-0.5 L_{y}\right), f_{i y k}^{p}=f_{i y k}\left(0.5 L_{y}\right), f_{i y k}^{a m}=\frac{\partial f_{i y k}}{\partial y}\left(-0.5 L_{y}\right), f_{i y k}^{a p}=\frac{\partial f_{i y k}}{\partial y}\left(0.5 L_{y}\right)$, $k=1,2$.

Then the initial-boundary value problem (3.2) can be written in the following form

$$
\left\{\begin{array}{l}
\frac{\partial c_{y}(x, t)}{\partial t}=\frac{\partial}{\partial x}\left(D_{x} \frac{\partial c_{y}(x, t)}{\partial x}\right)+r_{x} \frac{\partial c_{y}(x, t)}{\partial x}-a_{0}^{2} c_{y}(x, t) \\
\quad+\left(H_{y}^{m} B_{y}^{m}-H_{y}^{e} B_{y}^{e}\right)\left(c_{y}(x, t)-c_{a y}^{v}(x)\right)+\left(H_{z}^{e} B_{1 z}^{e}+H_{z}^{m} B_{1 z}^{m}\right) c_{y}(x, t) \\
\quad+\left(H_{z}^{e} B_{2 z}^{e}+H_{z}^{m} B_{2 z}^{m}\right) c_{0}^{v}(x)+f_{y}(x, t) \\
\frac{\partial c_{y}(0, t)}{\partial x}=0, D_{x} \frac{\partial c_{y}\left(L_{x}, t\right)}{\partial x}+\alpha_{x}\left(c_{y}\left(L_{x}, t\right)-c_{a x}^{v v}\right)=0 \\
c_{y}(x, 0)=c_{y, 0}(x), x \in\left(0, L_{x}\right), t \in\left(0, t_{f}\right)
\end{array}\right.
$$

where $H_{y}^{e}, H_{y}^{m}, B_{y}^{e}, B_{y}^{m}$ are the $N$-order diagonal matrices with the elements $h_{i y}^{e}=\frac{G_{i y}}{L_{y}}\left(D_{i y}\left(f_{i y 2}^{a p}-f_{i y 2}^{a m}\right)+r_{i y}\left(f_{i y 2}^{p}-f_{i y 2}^{m}\right)\right), h_{i y}^{m}=\frac{1}{L_{y}}\left(D_{i y}\left(f_{i y 1}^{a p}-f_{i y 1}^{a m}\right)+\right.$ $\left.r_{i y}\left(f_{i y 1}^{p}-f_{i y 1}^{m}\right)\right), b_{i y}^{e}=b_{i y}, b_{i y}^{m}=G_{i y} \kappa_{i y}^{m} b_{i y}, c_{y}, f_{y}$ are the $N$-order vectorscolumns with the elements $c_{i y}, f_{i y}, c_{0}^{v}(x)$ is the $N$-order vector-column with two nonzero elements $c_{o z}^{v}(x), c_{a z}^{v}(x), f_{y}(x, t)=\frac{1}{L_{y}} \int_{0}^{L_{y}} f_{z}(x, y, t) d y, c_{a z}^{v}(x)=$ $\frac{1}{L_{y}} \int_{0}^{L_{y}} c_{a z}(x, y) d y, c_{o z}^{v}(x)=\frac{1}{L_{y}} \int_{0}^{L_{y}} c_{o z}(x, y) d y, c_{a x}^{v v}=\frac{1}{L_{y}} \int_{0}^{L_{y}} c_{a x}^{v}(y) d y$, $c_{y, 0}(x)=\frac{1}{L_{y}} \int_{0}^{L_{y}} c_{z, 0}(x, y) d y$.

\section{The averaging method in $x$-direction}

It is also possible to do the averaging with respect to $x$ coordinate:

$$
\begin{aligned}
c_{i x}(t) & =\frac{1}{L_{x}} \int_{0}^{L_{x}} c_{i y}(x, t) d x, \quad c_{i y}(x, t)=c_{i x}(t)+m_{i x}(t) f_{i x 1}\left(x-0.5 L_{x}\right) \\
+ & e_{i x}(t) G_{i x} f_{i x 2}\left(x-0.5 L_{x}\right), \quad \int_{0}^{L_{x}} f_{i x 1} d y=\int_{0}^{L_{x}} f_{i x 2} d y=0,
\end{aligned}
$$

with the unknown functions $m_{i x}(t), e_{i x}(t)$. For example,

$$
\begin{aligned}
& f_{i x 1}=\exp \left(a_{i x 1}\left(x-0.5 L_{x}\right)\right)-\frac{2}{a_{i x 1} L_{x}} \sinh \left(0.5 a_{i x 1} L_{x}\right), \\
& f_{i x 2}=\exp \left(a_{i x 2}\left(x-0.5 L_{x}\right)\right)-\frac{2}{a_{i x 2} L_{x}} \sinh \left(0.5 a_{i x 2} L_{x}\right),
\end{aligned}
$$

where $a_{i x 1}=-\frac{r_{i x}}{2 D_{i x}}-\sqrt{\frac{r_{i x}^{2}}{4 D_{i x}^{2}}+\frac{k_{i x 0}^{2}}{D_{i x}}}, a_{i x 2}=-\frac{r_{i x}}{2 D_{i x}}+\sqrt{\frac{r_{i x}^{2}}{4 D_{i x}^{2}}+\frac{k_{i x 0}^{2}}{D_{i x}}}, k_{i x 0}^{2}=$ $a_{i 0}^{2}-a_{i x}, a_{i x} \leq 0$ is the coefficient depending on $c_{i y}$ in (4.1).

We can determine these functions from boundary conditions (4.1) in the following form:

$$
\begin{aligned}
& D_{i x}\left(m_{i x} f_{i x 1}^{a p}+e_{i x} G_{i x} f_{i x 2}^{a p}\right)+\alpha_{x}\left(m_{i x} f_{i x 1}^{p}+e_{i x} G_{i x} f_{i x 2}^{p}+c_{i x}(t)-c_{i a x}^{v v}\right)=0, \\
& m_{i x} f_{i x 1}^{a m}+e_{i x} G_{i x} f_{i x 2}^{a m}=0 \text { or } e_{i x}=-b_{i x}\left(c_{i x}(t)-c_{i a x}^{v v}\right), \quad m_{i x}=-G_{i x} \kappa_{i x}^{m} e_{i x},
\end{aligned}
$$


where $b_{i x}=\alpha_{i x} /\left(\alpha_{i x} G_{i x}\left(f_{i x 2}^{p}-\kappa_{i x}^{m} f_{i x 1}^{p}\right)+L_{x}\left(f_{i x 2}^{a p}-\kappa_{i x}^{m} f_{i x 1}^{a p}\right)\right), \kappa_{i x}^{m}=\frac{f_{i x 2}^{a m}}{f_{i x 1}^{a m}}$, $G_{i x}=\frac{L_{x}}{D_{i x}}, f_{i x k}^{m}=f_{i x k}\left(-0.5 L_{x}\right), f_{i x k}^{p}=f_{i x k}\left(0.5 L_{x}\right), f_{i x k}^{a m}=\frac{\partial f_{i x k}}{\partial x}\left(-0.5 L_{x}\right)$, $f_{i x k}^{a p}=\frac{\partial f_{i x k}}{\partial x}\left(0.5 L_{x}\right), k=1,2$.

Then the initial-boundary value problem (4.1) can be written in the following form:

$$
\left\{\begin{array}{l}
\frac{\partial c_{x}(t)}{\partial t}=\left(H_{y}^{m} B_{y}^{m}-H_{y}^{e} B_{y}^{e}\right)\left(c_{x}(t)-c_{a y}^{v v}\right)+\left(H_{z}^{e} B_{1 z}^{e}+H_{z}^{m} B_{1 z}^{m}\right) c_{x}(t)-a_{0}^{2} c_{x}(t) \\
\quad+\left(H_{z}^{e} B_{2 z}^{e}+H_{z}^{m} B_{2 z}^{m}\right) c_{0}^{v v}+\left(H_{x}^{m} B_{x}^{m}-H_{x}^{e} B_{x}^{e}\right)\left(c_{x}(t)-c_{a x}^{v v}\right)+f_{x}(t) \\
c_{x}(0)=c_{x, 0}, \quad t \in\left(0, t_{f}\right)
\end{array}\right.
$$

where $H_{x}^{e}, H_{x}^{m}, B_{x}^{e}, B_{x}^{m}$ are the $N$-order diagonal matrices with the elements $h_{i x}^{e}=\frac{G_{i x}}{L_{x}}\left(D_{i x}\left(f_{i x 2}^{a p}-f_{i x 2}^{a m}\right)+r_{i x}\left(f_{i x 2}^{p}-f_{i x 2}^{m}\right)\right), h_{i x}^{m}=\frac{1}{L_{x}}\left(D_{i x}\left(f_{i x 1}^{a p}-f_{i x 1}^{a m}\right)+\right.$ $\left.r_{i x}\left(f_{i x 1}^{p}-f_{i x 1}^{m}\right)\right), b_{i x}^{e}=b_{i x}, b_{i x}^{m}=G_{i x} \kappa_{i x}^{m} b_{i x}, c_{x}, f_{x}$ are the $N$-order vectorscolumns with elements $c_{i x}, f_{i x}, c_{0}^{v v}$, are the $N$-order vector-column with two nonzero elements $c_{o z}^{v v}, c_{a z}^{v v}$,

$$
\begin{aligned}
& f_{x}(t)=\frac{1}{L_{x}} \int_{0}^{L_{x}} f_{y}(x, t) d x, \quad c_{x, 0}=\frac{1}{L_{x}} \int_{0}^{L_{x}} c_{y, 0}(x) d x, \\
& c_{a z}^{v v}=\frac{1}{L_{x}} \int_{0}^{L_{x}} c_{a z}^{v}(x) d x, \quad c_{0 z}^{v v}=\frac{1}{L_{x}} \int_{0}^{L_{x}} c_{0 z}^{v}(x) d x .
\end{aligned}
$$

Therefore from (5.1) we have the initial value problem for the system of $N$ ODEs of the first order. The solutions for this system can be obtained with classical methods.

For the averaged stationary solution ( $f_{x}$ is a constant vector) we also get the analytical solution in the form

$$
c_{x}=\left(H_{x, y, z}\right)^{-1}\left(-f_{x}+g\right),
$$

where $H_{x, y, z}=H_{z}^{e} B_{1 z}^{e}+H_{z}^{m} B_{1 z}^{m}+H_{y}^{m} B_{y}^{m}-H_{y}^{e} B_{y}^{e}+H_{x}^{m} B_{x}^{m}-H_{x}^{e} B_{x}^{e}-a_{0}^{2}$, is the $N$-order matrix and $g=\left(H_{x}^{m} B_{x}^{m}-H_{x}^{e} B_{x}^{e}\right) c_{a x}^{v v}+\left(H_{y}^{m} B_{y}^{m}-H_{y}^{e} B_{y}^{e}\right) c_{a y}^{v v}-$ $\left(H_{z}^{e} B_{2 z}^{e}+H_{z}^{m} B_{2 z}^{m}\right) c_{0}^{v v}$ is the $N$-order vector-column.

\section{CAM for solving 1-D multi-layer diffusion problem with the hyperbolic type integral spline}

For solving (2.1) without convection $r_{i x}=r_{i y}=r_{i z}=0$ we consider hyperbolic functions in the $z$-direction with parameters $a_{i z 1}, a_{i z 2}, i=\overline{1, N}$ :

$$
f_{i z 1}=\frac{0.5 H_{i} \sinh \left(a_{i z 1}\left(z-\overline{z_{i}}\right)\right)}{\sinh \left(0.5 a_{i z 1} H_{i}\right)}, \quad f_{i z 2}=\left(0.25 \frac{\sinh ^{2}\left(a_{i z 2}\left(z-\overline{z_{i}}\right)\right)}{\sinh ^{2}\left(0.5 a_{i z 2} H_{i}\right.}-A_{i 0 z}\right),
$$

where $A_{i 0 z}=0.25 \frac{\left(\sinh \left(a_{i z 2} H_{i}\right)\right) /\left(a_{i z 2} H_{i}\right)-1}{\cosh \left(a_{i z 2} H_{i}\right)-1} \in[0,1 / 12]$. We see that the parameters $a_{i z 1}, a_{i z 2}$ tend to zero when the limit is the integral parabolic spline [4], because $A_{i 0 z} \rightarrow \frac{1}{12}$ as $f_{i z 1}=\left(z-\overline{z_{i}}\right), f_{i z 2}=\frac{\left(z-\overline{z_{i}}\right)^{2}}{H_{i}^{2}}-\frac{1}{12}$. We also see 
that $f_{i z 1}^{m}=-0.5 H_{i}, f_{i z 1}^{p}=0.5 H_{i}, f_{i z 2}^{m}=f_{i z 2}^{p}=A_{i 1 z}, f_{i z 1}^{a m}=f_{i z 1}^{a p}=d_{i z 1}$, $f_{i z 2}^{a m}=-d_{i z 2}, f_{i z 2}^{a p}=d_{i z 2}$, where $A_{i 1 z}=0.25-A_{i 0 z} \in[1 / 6,1 / 4], A_{i 1 z} \rightarrow \frac{1}{6}$, $d_{i z 1}=0.5 H_{i} a_{i z 1} \operatorname{coth}\left(0.5 a_{i z 1} H_{i}\right) \rightarrow 1, d_{i z 2}=0.5 H_{i} a_{i z 2} \operatorname{coth}\left(0.5 a_{i z 2} H_{i}\right) \rightarrow 1$, if $a_{i z 1}, a_{i z 2} \rightarrow 0$.

It is possible to show that this spline for $a_{i z 1}=\sqrt{a_{i 0}^{2} / D_{i z}}, a_{i z 2}=0.5 a_{i z 1}$ integrate exactly the following $1 \mathrm{D}$ boundary-value problem:

$$
\left\{\begin{array}{l}
\frac{\partial}{\partial z}\left(D_{i z} \frac{\partial c_{i}(z)}{\partial z}\right)-a_{i 0}^{2} c_{i}(z)+F_{i}=0, \quad z \in\left(z_{i-1}, z_{i}\right), i=\overline{1, N}, \\
D_{1 z} \frac{\partial c_{1}(0)}{\partial z}-\beta_{z}\left(c_{1}(0)-C_{0}\right)=0, \\
D_{N z} \frac{\partial c_{N}\left(L_{z}\right)}{\partial z}+\alpha_{z}\left(c_{N}\left(L_{z}\right)-C_{a}\right)=0, \\
c_{i}\left(z_{i}\right)=c_{i+1}\left(z_{i}\right), D_{i z} \frac{\partial c_{i}\left(z_{i}\right)}{\partial z}=D_{i+1, z} \frac{\partial c_{i+1}\left(z_{1}\right)}{\partial z}, \quad i=\overline{1, N-1},
\end{array}\right.
$$

where $F_{i}, C_{0}, C_{a}$ are constant parameter.

Table 1. The maximal error $\delta$ and averaged values $c_{a n}^{v}$-exact, $c_{a p}^{v}$-approx)

\begin{tabular}{lllllll}
\hline$\alpha_{z}$ & $\beta_{z}$ & $a_{1}$ & $a_{2}$ & $\delta$ & $c_{a p}^{v}$ & $c_{a n}^{v}$ \\
\hline 2 & 1 & 0 & 0 & 0.092 & 0.769 & 0.792 \\
- & - & 0.5 & 0.5 & 0.084 & 0.802 & - \\
- & - & 1.0 & 1.0 & 0.062 & 0.785 & - \\
- & - & 1.4 & 1.4 & 0.050 & 0.791 & - \\
- & - & 1.5 & 1.5 & 0.050 & 0.802 & - \\
- & - & $\mathrm{b}$ & $\mathrm{b} / 2$ & 0.0 & 0.792 & 0.792 \\
2 & 0 & 0 & 0 & 0.339 & 0.672 & 0.808 \\
- & - & 1.3 & 1.3 & 0.243 & 0.727 & - \\
- & - & 2.0 & 2.0 & 0.134 & 0.791 & - \\
- & - & 2.5 & 2.5 & 0.088 & 0.843 & - \\
- & - & 2.3 & 2.3 & 0.081 & 0.822 & - \\
- & - & $\mathrm{b}$ & $\mathrm{b} / 2$ & 0.0 & 0.808 & 0.808 \\
20 & 0 & 0 & 0 & 0.339 & 0.672 & 0.809 \\
- & - & 2.3 & 2.3 & 0.081 & 0.823 & - \\
- & - & $\mathrm{b}$ & $\mathrm{b} / 2$ & 0.0 & 0.809 & 0.809 \\
\hline
\end{tabular}

Example 1. We consider the 1-D problem (6.1) with one layer $(N=1)$ in the following form:

$$
\left\{\begin{array}{l}
\frac{\partial}{\partial z}\left(\frac{\partial c(z)}{\partial z}\right)-b^{2} c(z)+f_{0}=0, \quad z \in\left(0, L_{z}\right), \\
D_{z} \frac{\partial c(0)}{\partial z}-\beta_{z}\left(c(0)-C_{0}\right)=0, D_{z} \frac{\partial c\left(L_{z}\right)}{\partial z}+\alpha_{z}\left(c\left(L_{z}\right)-C_{a}\right)=0,
\end{array}\right.
$$

where $b^{2}=a_{0}^{2} / D_{z}, f_{0}=F / D_{z}$. Therefore the exact solution is

$$
c(z)=P_{1} \sinh (b z)+P_{2} \cosh (b z)+f_{0} / b^{2},
$$

where the constants

$$
\begin{aligned}
P_{1}= & \beta_{z}\left(P_{2}+f_{0} / b^{2} / D_{z} b-C_{0}\right), \quad P_{2}=\alpha_{z}\left(C_{a}-f_{0} / b^{2}\right)+\beta\left(C_{0}-f_{0} / b^{2}\right) \\
\times & \left(\cosh \left(b L_{z}\right)+\alpha_{z} \sinh \left(b L_{z}\right) /\left(b D_{z}\right)\right) /\left(\cosh \left(b L_{z}\right)\left(\alpha_{z}+\beta_{z}\right)\right. \\
& \left.+\sinh \left(b L_{z}\right)\left(b D_{z}+\alpha_{z} \beta_{z} /\left(b D_{z}\right)\right)\right) .
\end{aligned}
$$


The averaged values are $c^{v}=L_{z}^{-1} \int_{0}^{L_{z}} c(z) d z=\frac{1}{L_{z} b}\left(P_{1}\left(\cosh \left(b L_{z}\right)-1\right)+\right.$ $\left.P_{2} \sinh \left(b L_{z}\right)\right)+f_{0} / b^{2}$.

This form of solution was also applied to discreate approximation $c\left(z_{j}\right), z_{j}=$ $j h, h=\frac{L_{z}}{N}, j=\overline{0, N}$ by using the exact finite difference scheme (FDS) from N. Bahvalov [1]. For comparison we use the averaged method with respect to $z$ for hyperbolic type integral spline.

The Table 1 shows the numerical results for $F=0, L_{z}=1, L_{x}=1, L_{y}=$ $1, C_{0}=0.3, C_{a}=2.0, D_{z}=10^{-3}, D_{x}=D_{y}=3 \cdot 10^{-4}, b=2.4335$. The maximal error $\delta$ and averaged values are presented, the dependence on $a_{1}=a_{z 1}$, $a_{2}=a_{z 2}, \alpha_{z}, \beta_{z}\left(a_{1}=a_{2}=0\right.$ for parabolic spline $)$ is investigated.

Figures 1, 2 represent the solutions $c(z)$ for $N=20, \alpha_{z}=20, \beta_{z}=0$, $a_{1}=a_{2}=2.3$ and for $a_{1}=b, a_{2}=b / 2$, respectively. Note that for $a_{1}=a_{2}$ and FDS we get $\delta=0.0222$, and for $a_{1}=b, a_{2}=b / 2$ we get $\delta=0$. For $L_{z}=3, F=0.1, b=2.4335$ follows: $c_{a p}^{v}=23.14, c_{a n}^{v}=16.87, \delta=6.27$ (for parabolic spline), $c_{a p}^{v}=16.87, c_{a n}^{v}=16.87, \delta=0$ (for hyperbolic type spline with $a_{1}=b, a_{2}=b / 2$ ).

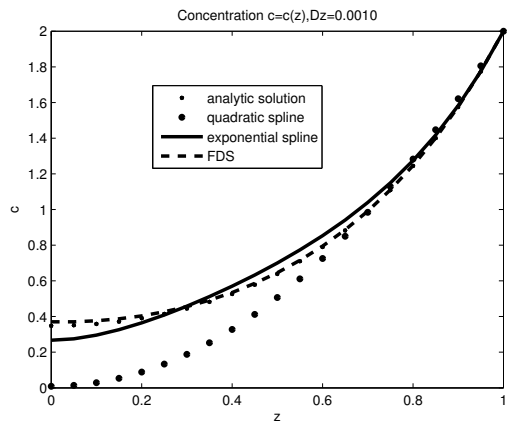

Figure 1. One layer solution $\mathrm{c}(\mathrm{z})$ for $L_{z}=1, \alpha_{z}=20, \beta_{z}=0, a_{1}=a_{2}=2.3$

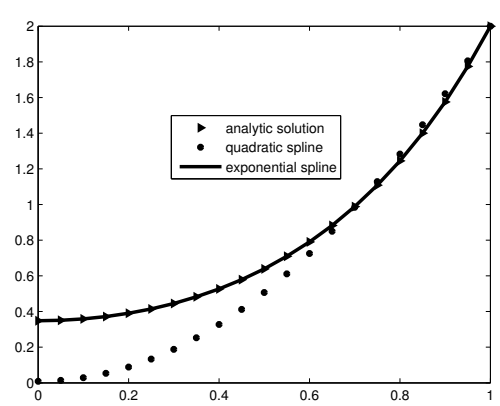

Figure 2. One layer solution $c(z)$ for $L_{z}=$ $3, D_{1 z}=10 e-03, D_{2 z}=10 e-04, a_{2}=a_{1} / 2$

Figures 3, 4 represent the functions $f_{1 z}, f_{2 z}$ for the hyperbolic spline depending on parameters $a_{1}=a_{2} \in[0,25]$, and for the parabolic spline $a_{1}=a_{2}=0$. For the spline with 2 parameters $a_{1}=b, a_{2}=b / 2$ we have the exact solution for all values of parameters $L_{z}, F, b, D_{z}, C_{0}, C_{a}, \alpha, \beta$.

\section{CAM for solving 1-D multi-layer mass transfer problem with the exponential type integral spline}

It is possible to show that the integral spline integrates the exact following 1D boundary-value problem in $N$ layers:

$$
\left\{\begin{array}{l}
\frac{\partial}{\partial z}\left(D_{i z} \frac{\partial c_{i}(z)}{\partial z}\right)+r_{i z} \frac{\partial c_{i}(z)}{\partial z}-a_{i 0}^{2} c_{i}(z)+F_{i}=0, z \in\left(z_{i-1}, z_{i}\right), i=\overline{1, N} \\
D_{1 z} \frac{\partial c_{1}(0)}{\partial z}-\beta_{z}\left(c_{1}(0)-C_{0}\right)=0 \\
D_{N z} \frac{\partial c_{N}\left(L_{z}\right)}{\partial z}+\alpha_{z}\left(c_{N}\left(L_{z}\right)-C_{a}\right)=0 \\
c_{i}\left(z_{i}\right)=c_{i+1}\left(z_{i}\right), D_{i z} \frac{\partial c_{i}\left(z_{i}\right)}{\partial z}=D_{i+1, z} \frac{\partial c_{i+1}\left(z_{1}\right)}{\partial z}, i=\overline{1, N-1}
\end{array}\right.
$$




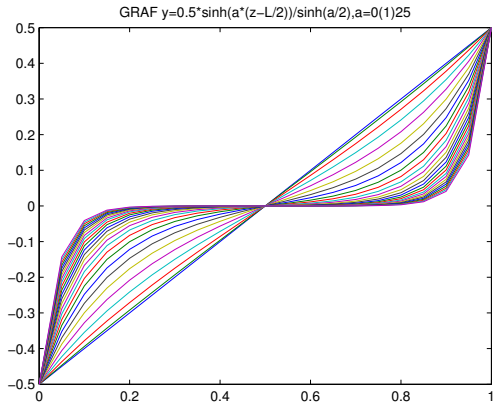

Figure 3. Values of functions $f_{1 z}$ for hyperbolic spline with $a_{1}=a_{2} \in[0,25]$

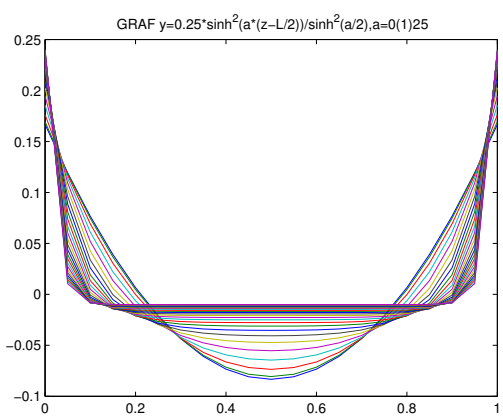

Figure 4. Values of functions $f_{2 z}$ for hyperbolic spline with $a_{1}=a_{2} \in[0,25]$

where $F_{i}, C_{0}, C_{a}$ are constant parameters (see Section $10(N=5$ and $N=10)$ ).

Using the averaging in $z$-direction $(3.1,3.2)$ we have the averaged value $c_{z}^{v}$ for the solution $c_{i}(z)$ of boundary-value problem (7.1) in the following matrix form:

$$
c_{z}^{v}=\left(a_{02}-H_{z}^{e} B_{1 z}^{e}-H_{z}^{m} B_{1 z}^{m}\right)^{-1}\left(\left(H_{z}^{e} B_{2 z}^{e}+H_{z}^{m} B_{2 z}^{m}\right) c_{0}+F\right),
$$

where the elements on the diagonal of $N$-order matrices $H_{z}^{e}, H_{z}^{m}$ are $h_{i z}^{e}=$ $\frac{2 G_{z}}{H_{i}}\left(D_{i z} a_{i z 2}+r_{i z}\right) \sinh \left(0.5 a_{i z 2} H_{i}\right)$ and $h_{i z}^{m}=\frac{2}{H_{i}}\left(D_{i z} a_{i z 1}+r_{i z}\right) \sinh \left(0.5 a_{i z 1} H_{i}\right)$.

The spline-solution is defined as:

$$
c(z)=c_{z}^{v}+F_{z 1} m_{z}+G_{z} F_{z 2} e_{z}
$$

with $N$-order diagonal matrices $F_{z 1}, F_{z 2}, G_{z}$. Similarly the exact analytical solution of (7.1) can be obtained.

For two layers $(N=2)$ by $L_{z}=3, H_{1}=1.8, H_{2}=1.2, D_{1}=10^{-3}, D_{2}=$ $10^{-4}, \alpha_{1}=10^{6}, \beta_{1}=10^{3}, C_{0}=0.3, C_{a}=3.0, r_{1}=-0.01, r_{2}=0.01, F_{1}=$ $0.001, F_{2}=0.001$, we have the following numerical results (see Figure 5): the maximal error $-2 \cdot 10^{-7}$ (exponential spline), 0.6045 (parabolic spline), the averaged values $c_{1}^{v}=0.3332, c_{2}^{v}=0.8391$ (exact and exponential splines), $c_{1}^{v}=0.3662, c_{2}^{v}=0.8055$ (parabolic spline). Figure 6 represents how function $f_{1 z 1}$ for exponential spline is depending on the parameter $a 1_{1 z} \in[-5,5]$.

\section{CAM for solving 3-D diffusion problem in one layer with the exponential type integral spline}

For averaging (2.1) in one layer $\left(\overline{z_{1}}=L_{z} / 2\right)$ we consider exponential functions following in the $z$-direction. From boundary conditions the system of two algebraic equations follows

$$
\left\{\begin{array}{l}
b_{11} m_{z}+b_{12} e_{z}=\beta_{z}\left(c_{z}-c_{0 z}\right), \\
b_{21} m_{z}+b_{22} e_{z}=-\alpha_{z}\left(c_{z}-c_{a z}\right),
\end{array}\right.
$$




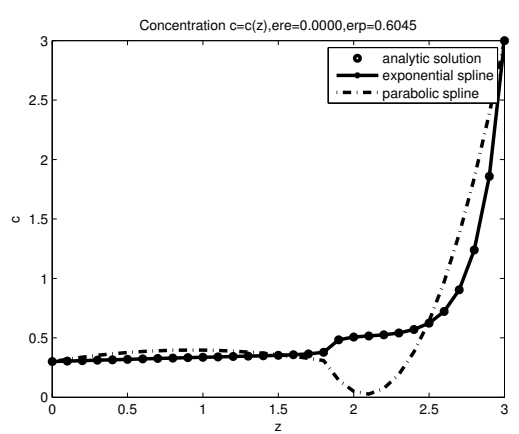

Figure 5. Two layers solutions for $L_{z}=3, D_{1 z}=10 e-03, D_{2 z}=10 e-04$

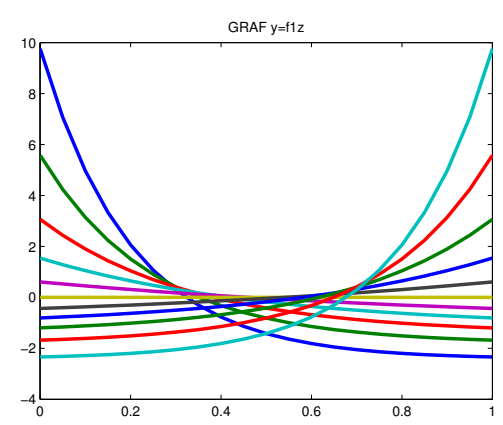

Figure 6. Values of functions $f_{1 z 1}$ for exponential spline, $a 1_{1 z} \in[-5(1) 5]$

where $b_{11}=D_{z} f_{z 1}^{a m}-\beta_{z} f_{z 1}^{m}, b_{21}=D_{z} f_{z 1}^{a p}+\alpha_{z} f_{z 1}^{p}, b_{12}=L_{z} f_{z 2}^{a m}-G_{z} \beta_{z} f_{z 2}^{m}$, $b_{22}=L_{z} f_{z 2}^{a p}+G_{z} \alpha_{z} f_{z 2}^{p}$. The solution is given in the form

$$
m_{z}=a_{m} c_{z}-b_{m} c_{a z}-c_{m} c_{0 z}, \quad e_{z}=-a_{e} c_{z}-b_{e} c_{a z}+c_{e} c_{0 z},
$$

where $a_{m}=\left(\beta_{z} b_{22}+\alpha_{z} b_{12}\right) / d, b_{m}=\alpha_{z} b_{12} / d, c_{m}=\beta_{z} b_{22} / d, a_{e}=\left(\alpha_{z} b_{11}+\right.$ $\left.\beta_{z} b_{21}\right) / d, b_{e}=\alpha_{z} b_{11} / d, c_{e}=\beta_{z} b_{21} / d, d=b_{11} b_{22}-b_{12} b_{21}$.

The new 2D initial-boundary value problem is in the form

$$
\left\{\begin{array}{l}
\frac{\partial c_{z}(x, y, t)}{\partial t}=\frac{\partial}{\partial x}\left(D_{x} \frac{\partial c_{z}(x, y, t)}{\partial x}\right)+r_{x} \frac{\partial c_{z}(x, y, t)}{\partial x} \\
\quad+\frac{\partial}{\partial y}\left(D_{y} \frac{\partial c_{z}(x, y, t)}{\partial y}\right)+r_{y} \frac{\partial c_{z}(x, y, t)}{\partial y}+\left(g_{z}-a_{0}^{2}\right) c_{i z}(x, y, t) \\
\quad+a_{z} c_{a z}+b_{z} c_{0 z}+f_{z}(x, y, t), \\
\frac{\partial c_{z}(0, y, t)}{\partial x}=\frac{\partial c_{z}(x, 0, t)}{\partial y}=0, \\
D_{x} \frac{\partial c_{z}\left(L_{x}, y, t\right)}{\partial x}+\alpha_{x}\left(c_{z}\left(L_{x}, y, t\right)-c_{a x}^{v}(y)\right)=0 \\
D_{y} \frac{\partial c_{z}\left(x, L_{y}, t\right)}{\partial y}+\alpha_{y}\left(c_{z}\left(x, L_{y}, t\right)-c_{a y}^{v}(x)\right)=0, \\
c_{z}(x, y, 0)=c_{z, 0}(x, y), \quad x \in\left(0, L_{x}\right), y \in\left(0, L_{y}\right), t \in\left(0, t_{f}\right),
\end{array}\right.
$$

where $g_{z}=b_{1} a_{m}-b_{2} G_{z} a_{e} \leq 0, a_{z}=-b_{1} b_{m}+b_{1} G_{z} b_{e}, b_{z}=-b_{1} c_{m}+b_{2} G_{z} c_{e}$, $b_{1}=\frac{2}{L_{z}} \sinh \left(a_{z 1} L_{z} / 2\right)\left(D_{z} a_{z 1}+r_{z}\right), b_{2}=\frac{2}{L_{z}} \sinh \left(a_{z 2} L_{z} / 2\right)\left(D_{z} a_{z 2}+r_{z}\right)$.

For averaging in the $y$-direction we use the following parameters:

$$
a_{y 1}=-\frac{r_{y}}{2 D_{y}}-\sqrt{\frac{r_{y}^{2}}{4 D_{y}^{2}}+\frac{\left(a_{0}^{2}-g_{z}\right)}{D_{y}}}, \quad a_{y 2}=-\frac{r_{y}}{2 D_{y}}+\sqrt{\frac{r_{y}^{2}}{4 D_{y}^{2}}+\frac{\left(a_{0}^{2}-g_{z}\right)}{D_{y}}} .
$$

We see that $f_{y k}^{p}=d_{k p y}, f_{y k}^{a m}=a_{y k} \exp \left(-0.5 a_{y k} L_{y}\right), f_{y k}^{a p}=a_{y k} \exp \left(0.5 a_{y k} L_{y}\right)$, $f_{y k}^{p}=\exp \left(0.5 a_{y k} L_{y}\right)-\frac{2}{a_{y k} L_{y}} \sinh \left(0.5 a_{y k} L_{y}\right), k=1,2$.

It follows from boundary conditions that

$$
m_{y}=-e_{y} G_{y} \kappa_{y}, \quad e_{y}=-b_{y}\left(c_{y}-c_{a y}^{v}\right),
$$

where $\kappa_{y}=f_{y 2}^{a m} / f_{y 1}^{a m}, b_{y}=\alpha_{y} /\left(L_{y}\left(f_{y 2}^{a p}-\kappa_{y} f_{y 1}^{a p}\right)+\alpha_{y} G_{y}\left(f_{y 2}^{p}-\kappa_{y} f_{y 1}^{p}\right)\right)$. 
The new 1D initial-boundary value problem is in the form

$$
\left\{\begin{array}{l}
\frac{\partial c_{y}(x, t)}{\partial t}=\frac{\partial}{\partial x}\left(D_{x} \frac{\partial c_{y}(x, t)}{\partial x}\right)+r_{x} \frac{\partial c_{z}(x, t)}{\partial x} \\
\quad+\left(g_{z}-a_{0}^{2}-b_{y} g_{y}\right) c_{y}(x, t)+a_{z} c_{a z}^{v}+b_{z} c_{0 z}^{v}+b_{y} g_{y} c_{a y}^{v}+f_{y}(x, t), \\
\frac{\partial c_{y}(0, t)}{\partial x}=0, D_{x} \frac{\partial c_{y}\left(L_{x}, t\right)}{\partial x}+\alpha_{x}\left(c_{y}\left(L_{x}, t\right)-c_{a x}^{v v}\right)=0 \\
c_{y}(x, 0)=c_{y, 0}(x), \quad x \in\left(0, L_{x}\right), \quad t \in\left(0, t_{f}\right),
\end{array}\right.
$$

where $g_{y}=f_{y 2}^{a p}-\kappa_{y} f_{y 1}^{a p}+\frac{2 r_{y}}{D_{y}}\left(-\kappa_{y} \sinh \left(a_{y 1} L_{y} / 2\right)+\sinh \left(a_{y 2} L_{y} / 2\right)\right) \geq 0$.

For averaging in the $x$-direction we use $a_{x 1}=-\frac{r_{x}}{2 D_{x}}-\sqrt{\frac{r_{x}^{2}}{4 D_{x}^{2}}+\frac{\left(a_{0}^{2}-g_{z}+b_{y} g_{y}\right)}{D_{x}}}$, $a_{x 2}=-\frac{r_{x}}{2 D_{x}}+\sqrt{\frac{r_{x}^{2}}{4 D_{x}^{2}}+\frac{\left(a_{0}^{2}-g_{z}+b_{y} g_{y}\right)}{D_{x}}}$. Then we get $f_{x k}^{p}=\exp \left(0.5 a k_{x} L_{x}\right)-$ $\frac{2}{a k_{x} L_{x}} \sinh \left(0.5 a k_{x} L_{x}\right), f_{x k}^{a m}=a k_{x} \exp \left(-0.5 a k_{x} L_{x}\right), f_{x k}^{a p}=a k_{x} \exp \left(0.5 a k_{x} L_{x}\right)$, $k=1,2$.

From boundary conditions it follows that

$$
m_{x}=-e_{x} G_{x} \kappa_{x}, \quad e_{x}=-b_{x}\left(c_{x}-c_{a x}^{v v}\right),
$$

where $\kappa_{x}=f_{x 2}^{a m} / f_{x 1}^{a m}, b_{x}=\alpha_{x} /\left(L_{x}\left(f_{x 2}^{a p}-\kappa_{x} f_{x 1}^{a p}\right)+\alpha_{x} G_{x}\left(f_{x 2}^{p}-\kappa_{x} f_{x 1}^{p}\right)\right)$.

We have the following initial value problem for ODEs of the first order:

$$
\left\{\begin{array}{l}
\frac{\partial c_{x}(t)}{\partial t}=\left(g_{z}-a_{0}^{2}-b_{y} g_{y}-b_{x} g_{x}\right) c_{x}(t) \\
\quad+a_{z} c_{a z}^{v v}+b_{z} c_{0 z}^{v v}+b_{y} g_{y} c_{a y}^{v v}+b_{x} g_{x} c_{a x}^{v v}+f_{x}(t) \\
c_{x}(0)=c_{x, 0}, \quad t \in\left(0, t_{f}\right)
\end{array}\right.
$$

where $g_{x}=f_{x 2}^{a p}-\kappa_{x} f_{x 1}^{a p}+\frac{2 r_{x}}{D_{x}}\left(-\kappa_{x} \sinh \left(a_{x 1} L_{x} / 2\right)+\sinh \left(a_{x 2} L_{x} / 2\right)\right) \geq 0$.

For the stationary solution we get

$$
c_{x}=\frac{a_{z} c_{a z}^{v v}+b_{z} c_{0 z}^{v v}+b_{y} g_{y} c_{a y}^{v v}+b_{x} g_{x} c_{a x}^{v v}+f_{x}}{-g_{z}+a_{0}^{2}+b_{y} g_{y}+b_{x} g_{x}} .
$$

Then we have the stationary 3D solution in following analytical form:

$c_{y}(x)=c_{x}+m_{x} f_{x 1}+e_{x} G_{x} f_{x 2}, e_{y}(x)=-b_{y}\left(c_{y}(x)-c_{a y}^{v}\right), m_{y}(x)=-e_{y}(x) G_{y} \kappa_{y}$, $c_{z}(x, y)=c_{y}(x)+m_{y}(x) f_{y 1}(y)+e_{y}(x) G_{y} f_{y 2}(y)$,

$e_{z}(x, y)=-a_{e} c_{z}(x, y)-b_{e} c_{a z}+c_{e} c_{0 z}, m_{z}(x, y)=a_{m} c_{z}(x, y)-b_{m} c_{a z}-c_{m} c_{0 z}$, $c(x, y, z)=c_{z}(x, y)+m_{z}(x, y) f_{z 1}(z)+e_{z}(x, y) G_{z} f_{z 2}(z)$.

\section{The numerical approximations with ADI method for the $3-\mathrm{D}$ problem in one layer}

We use a uniform space grid $(K+1) \times(\tilde{N}+1) \times(M+1)$ :

$$
\begin{gathered}
\left\{\left(z_{k}, y_{i}, x_{j}\right), \quad z_{k}=(k-1) h_{z}, \quad y_{i}=(i-1) h_{y}, \quad x_{j}=(j-1) h_{x}, \quad i=\overline{1, \tilde{N}+1}\right. \\
\left.j=\overline{1, M+1}, \quad k=\overline{1, K+1}, \quad K h_{z}=L_{z}, \tilde{N}, \quad h_{y}=L_{y}, \quad M h_{x}=L_{x}\right\} .
\end{gathered}
$$

For the time $t$ we use the discrete grid $t_{n}=n \tau, n=0,1, \ldots$. Subscripts $(k, i, j, n)$ refer to $z, y, x, t$ indices with the grid spacing, and for the approximation of function $c(z, y, x, t)$ we get a grid function $U_{k, i, j}^{n} \approx c\left(z_{k}, y_{i}, x_{j}, t_{n}\right)$. 
For solving 3D problem (2.1) we use the discrete approximation

$$
\left(U_{k, i, j}^{n+1}-U_{k, i, j}^{n}\right) / \tau=\left(\Lambda_{z}+\Lambda_{y}+\Lambda_{x}\right) U_{k, i, j}^{n+1}+f_{k, i, j}^{n}, \quad n \geq 0,
$$

$k=\overline{1, K+1}, i=\overline{1, \tilde{N}+1}, j=\overline{1, M+1}$ and ADI method by Douglas and Rachford [7,9]:

$$
\left\{\begin{array}{l}
\left(U_{k, i, j}^{n+1 / 3}-U_{k, i, j}^{n}\right) / \tau=\Lambda_{z} U_{k, i, j}^{n+1 / 3}+\Lambda_{y} U_{k, i, j}^{n}+\Lambda_{x} U_{i, j}^{n}+f_{i, j}^{n}, \\
k=\overline{2, K}, \quad i=2, \tilde{N}, \quad j=\overline{2, M} \\
\left(U_{k, i, j}^{n+2 / 3}-U_{k, i, j}^{n+1 / 3}\right) / \tau=\Lambda_{y}\left(U_{k, i, j}^{n+2 / 3}-U_{k, i, j}^{n}\right) \\
k=\overline{1, K+1}, \quad i=\overline{2, \tilde{N}}, \quad j=\overline{1, M+1} \\
\left(U_{k, i, j}^{n+1}-U_{k, i, j}^{n+2 / 3}\right) / \tau=\Lambda_{x}\left(U_{k, i, j}^{n+1}-U_{k, i, j}^{n}\right) \\
k=\overline{1, K+1}, \quad i=\overline{1, \tilde{N}+1}, \quad j=\overline{2, M}
\end{array}\right.
$$

After elimating the unknowns at fractional time moments $t_{n+1 / 3}, t_{n+2 / 3}$ we obtain the previous discrete problem with approximation error $O\left(\tau^{2}\right)$. Here $\Lambda_{x}, \Lambda_{y}, \Lambda_{z}$ are the difference operators, approximated the differential operators

$$
\begin{aligned}
& \frac{\partial}{\partial x}\left(D_{x} \frac{\partial c(z, y, x, t)}{\partial x}\right)+r_{x} \frac{\partial c(z, y, x, t)}{\partial x}, \frac{\partial}{\partial y}\left(D_{y} \frac{\partial c(z, y, x, t)}{\partial y}\right)+r_{y} \frac{\partial c(z, y, x, t)}{\partial y} \\
& \frac{\partial}{\partial z}\left(D_{z} \frac{\partial c(z, y, x, t)}{\partial z}\right)+r_{z} \frac{\partial c(z, y, x, t)}{\partial z}-a_{0}^{2} c(z, y, x, t)
\end{aligned}
$$

and boundary conditions with central differences, $f_{k, i, j}^{n}=f_{z}\left(z_{k}, y_{i}, x_{j}, t_{n}\right)$. The approximation is monotonous if $h_{x} \leq \frac{2 D_{x}}{\left|r_{x}\right|}, h_{y} \leq \frac{2 D_{y}}{\left|r_{y}\right|}, h_{z} \leq \frac{2 D_{z}}{\left|r_{z}\right|}$. For solving $U^{n+1 / 3}, U^{n+2 / 3}$ and $U^{n+1}$ we use Thomas algorithm in $z, y$ and $x$ directions respectively.

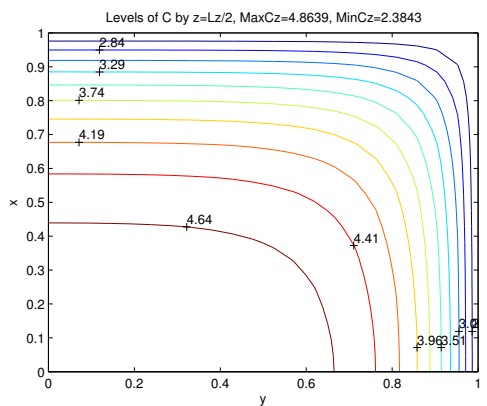

Figure 7. Levels of averaged concentration $c\left(x, y, L_{z} / 2\right)$

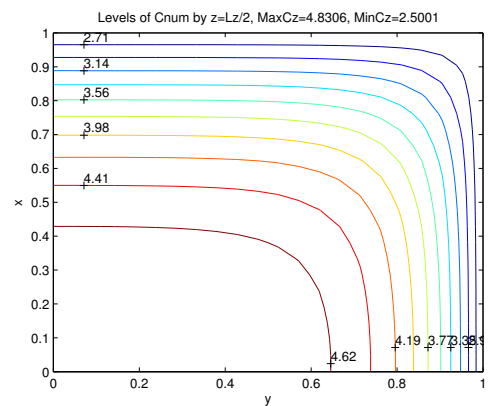

Figure 8. Levels of numerical concentration $c\left(x, y, L_{z} / 2\right)$

\section{Numerical results}

The numerical results are obtained for $K=19, \tilde{N}=M=21, L_{z}=3, L_{x}=$ $L_{y}=1, \alpha_{z}=20, \beta_{z}=10, \alpha_{x}=\alpha_{y}=2, c_{a z}=4, c_{0 z}=1.3, c_{a x}=c_{a y}=2.5$, 
$D_{x}=D_{y}=3 \cdot 10^{-3}, D_{z}=10^{-2}, r_{z}=0.01, r_{x}=0.001, r_{y}=-0.001, f=$ $0.1, a_{0}=0.1$. We have following numerical values: $g_{z}=-0.069, b_{y} g_{y}=$ $0.0129, b_{x} g_{x}=0.0281, a 1_{z}=-10.1, a 2_{z}=0.1, a 1_{y}=-3.73, a 2_{y}=7.06, a 1_{x}=$ $-7.45, a 2_{x}=3.73$.

For using the ADI method for the initial condition the stationary averaged solution $c_{z}(x, y, z)$ is selected. We have the stationary solution with $\tau=1$, $t_{f}=300$, then the maximal error is $10^{-8}$, the maximal value of $c(x, y, z)$ is 5.29 for averaged method, 5.16 for ADI method (see Figures 7-10).

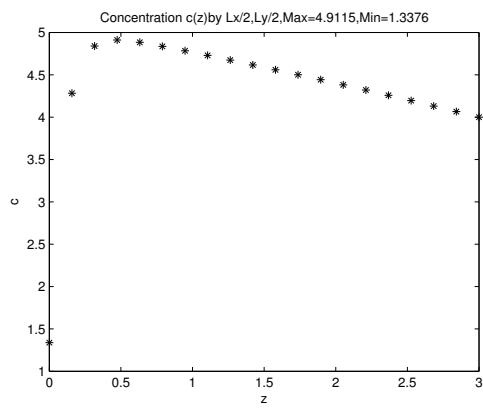

Figure 9. Averaged and numerical concentration $c\left(L_{x} / 2, L_{y} / 2, z\right)$

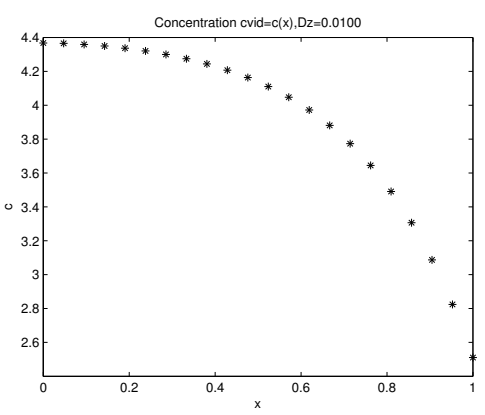

Figure 10. Averaged concentration $c_{x}(x)$

Depending on the number of grid points $(K, \tilde{N}, M)$ we have the following maximal values of the solution for ADI method: 5.1616 ( $K=19, M=\tilde{N}=$ 21), $5.1587(K=29, M=\tilde{N}=31)$.

Figure 11 shows the graphics of functions $c_{i}(z)$ (7.1) in 5 uniform layers $\left(N=5, H_{i}=0.6, L_{z}=3, \beta_{z}=\alpha_{z}=10, C_{0}=0.3, C_{a}=2\right)$ for $D_{i z}=$ $[0.01 ; 2 ; 0.03 ; 4 ; 0.05], r_{i z}=[0.1 ; 0.2 ; 0.3 ; 0.4 ; 0.5], F_{i}=[0.04 ; 0.08 ; 0.12 ; 0.16 ; 0.20]$, $a_{i 0}^{2}=[0.1 ; 0.2 ; 0.3 ; 0.4 ; 0.5]$. In Figure 12 the parameters remain the same, only the source term $F_{i}$ has the opposite sign.

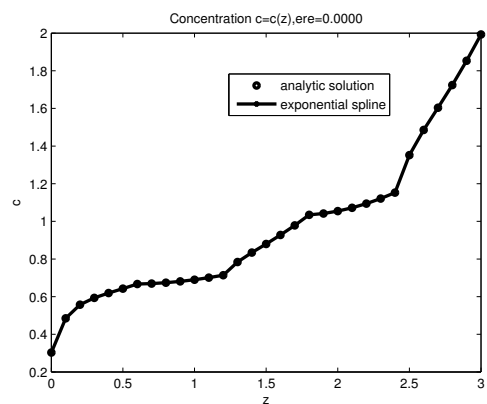

Figure 11. Function $c(z)$ for a positive source $\mathrm{F}(\mathrm{N}=5)$

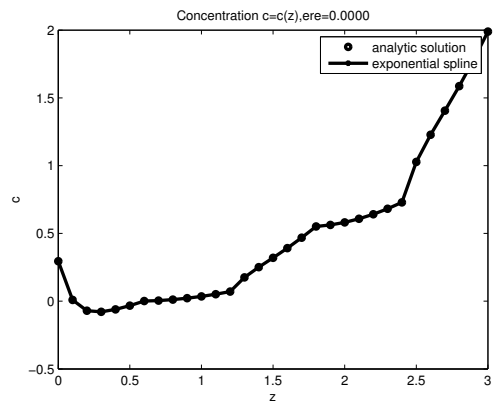

Figure 12. Function $c(z)$ for a negative source $\mathrm{F}(\mathrm{N}=5)$

Figure 13 shows the graphics of functions $c_{i}(z)(7.1)$ in 10 uniform layers 


$$
\begin{aligned}
\left(N=10, H_{i}\right. & \left.=0.6, L_{z}=6, \beta_{z}=\alpha_{z}=10, C_{0}=0.3, C_{a}=2\right) \text { for } \\
D_{i z} & =[0.01 ; 2 ; 0.03 ; 4 ; 0.05 ; 0.01 ; 2 ; 0.03 ; 4 ; 0.05], \\
r_{i z} & =[0.1 ; 0.2 ; 0.3 ; 0.4 ; 0.5 ; 0.1 ; 0.2 ; 0.3 ; 0.4 ; 0.5], \\
F_{i} & =[0.04 ; 0.08 ; 0.12 ; 0.16 ; 0.20 ; 0.04 ; 0.08 ; 0.12 ; 0.16 ; 0.20], \\
a_{i 0}^{2} & =[0.1 ; 0.2 ; 0.3 ; 0.4 ; 0.5 ; 0.1 ; 0.2 ; 0.3 ; 0.4 ; 0.5] .
\end{aligned}
$$

In Figure 14 the parameters remain the same as in Figure 13, but the source term $F_{i}$ has an opposite sign.

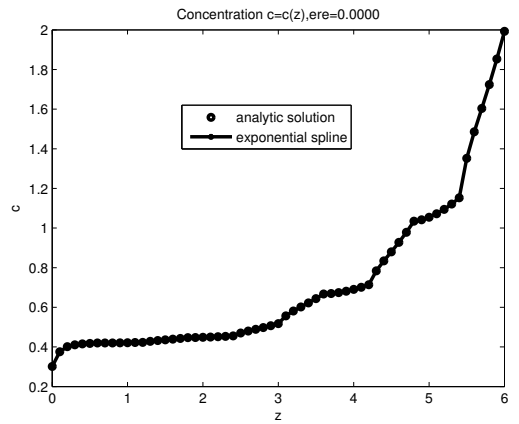

Figure 13. Function $c(z)$ for a positive source $\mathrm{F}(\mathrm{N}=10)$

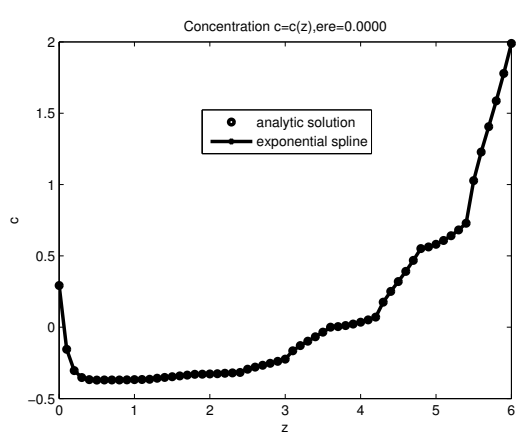

Figure 14. Function $c(z)$ for a negative source $\mathrm{F}(\mathrm{N}=10)$

\section{Conclusions}

The 3-D mass transfer problem in multi-layered domain is reduced to 2-D and 1-D problems using the special integral parabolic, hyperbolic and exponential type splines. These splines are obtained from the general spline with two fixed functions. The parameters of these functions are the characteristic values for the corresponding homogeneous ODEs of second order in fixed direction. These parameters are the best parameters for minimal error.

The 1-D differential and discrete problems are solved analytically. For hyperbolic spline the best parameter for minimal error is calculated.

The solutions for the corresponding averaged non-stationary 3-D initialboundary value problem are obtained numerically using alternating-direction implicit (ADI) method proposed by Douglas and Rachford.

The stationary numerical solution is compared with the analytical solution. The max absolute value of difference between corresponding numerical and averaged data was $1-2$ percent.

\section{Acknowledgement}

This work was partially supported by the grant $623 / 2014$ of the Latvian Council of Science. 


\section{References}

[1] N.S. Bahvalov, N.P.Zhitkov and G.M. Kobelhkov. Numerical methods. M, Nauka (in Russian), 1987.

[2] M. Buike, A. Buikis and H. Kalis. Time direct and time inverse problems for wave energy and steel quenching models, solved exactly and approximately. WSEAS Transactions on Heat and Mass Transfer, 10:30-43, 2015.

[3] A. Buikis. Modelling of Filtration Processes in Layered Porous Media by the Conservative Averaging Method. Kazan, 374 p., Dr. Thesis (in Russian), 1987.

[4] A. Buikis. Definition and calculation of a generalized integral parabolic spline. Proceedings of the Latvian Academy of Sciences. Section B, 7-8(576-577):97-100, 1995.

[5] R. Čiegis, G. Jankevičiūtė and O. Suboč. Numerical solution of the heat conductions in composite materials. Mathematical Modelling and Analysis, 15(1):9-22, 2010. http://dx.doi.org/10.3846/1392-6292.2010.15.9-22.

[6] R. Čiegis and N. Tumanova. Stability analysis of finite difference schemes for pseudoparabolic problems with nonlocal boundary conditions. Mathematical Modelling and Analysis, 19(2):285-297, 2014. http://dx.doi.org/10.3846/13926292.2014.910562.

[7] J. Douglas and H. H. Rachford. On the numerical solution of heat conduction problems in two and three space variables. Transactions of the American Mathematical Society, 82:421-439, 1956. http://dx.doi.org/10.1090/S0002-99471956-0084194-4.

[8] F.P. Incropera, D.P. DeWitt, Th.L. Bergman and A.S. Lavine. Fundamentals of heat and mass transfer, 6-th edition. John Wiley \& Sons, Hoboken(NJ), 2006.

[9] M. Sapagovas, G. Kairytè, O. Štikonienè and A. Štikonas. Alternating direction method for a two-dimensional parabolic equation with a nonlocal boundary condition. Mathematical Modelling and Analysis, 12(1):131-142, 2007. http://dx.doi.org/10.3846/1392-6292.2007.12.131-142.

[10] Vadimas Starikovičius, R. Čiegis and A. Bugajev. On efficiency analysis of the openfoam-based parallel solver for simulation of electrical power cables. Informatica, 27(1):161-178, 2016. http://dx.doi.org/10.15388/Informatica.2016.80.

[11] R. Vilums and A. Buikis. Conservative averaging method for partial differential equations with discontinuous coefficients. WSEAS Transactions on Heat and Mass Transfer, 1(4):383-390, 2006. 